\title{
Design and Thermal Simulation of Induction Machines for Traction in Electric and Hybrid Electric Vehicles
}

\begin{abstract}
Christian Kral*, Anton Haumer*, Hansjörg Kapeller*, Franz Pirker*
An electric traction machine for an electric or a hybrid electric vehicle is usually designed for a specific operating point or cycle. For such an operating point or cycle, the masses and the cooling circuit of the electric machine determine the time dependent temperature distribution within the machine. For a specific load cycle, the thermal simulation of the machine can reveal possible mass and size reductions for a given insulation class of the machine. In addition, such simulations allow the comparison of various cooling concepts. In the machine design process, the first step is a conventional electromagnetic machine design. From the geometric data of this design and the material properties, the parameters of a thermal equivalent circuit can be derived. The differential and algebraic equations of the thermal equivalent circuit are solved by a simulation tool to predict the temperatures of the critical parts in the electric machine. A thermal equivalent circuit is accurate enough to predict the thermal behavior of the critical parts in the electric machine, and yet not too complex, to obtain simulation results with moderate numerical effort. This enables an iterative design process to optimize the drive.
\end{abstract}

Keywords: Induction Motor, Electric Drive, Modeling, Simulation, Thermal Management

\section{MACHINE DESIGN PROCESS}

For the machine to be designed, a certain operating point or cycle has to be specified. The specifications of such an operating point or cycle could be the nominal voltage, frequency and torque, or nominal torque and speed including a voltage/frequency characteristic of the power inverter. In some cases the overload characteristic during acceleration is specified, too. An additional criteria for the electric machine design is to keep the size of the machine, and therefore cost of material as low as possible. The size of the machine is thereby significantly determined by the thermal and electromagnetic utilization with respect to the insulation class.

The electromagnetic design is based on the geometric data of the iron core and the windings of the stator and rotor $[1,2]$ of the electric machine. For determining the main geometric data, an initial estimation is performed using some characteristic parameters and experimental knowledge. An electromagnetic calculation software then calculates the magnetic and electromechanical characteristics for the specified operating point or cycle of the induction machine. For this calculation, the non linear iron properties and the estimation of the stray fluxes have to be taken into account. As a result of the calculated magnetic circuit, the magnetic characteristic of the machine is obtained. Some geometric parameters can then be tuned to performed and optimize the electromagnetic utilization considering the actual operating point or cycle. From the geometric data and the material properties, the ohmic resis-

*Arsenal Research, Giefinggasse 2, 1210 Vienna, Austria, email: christian.kral@arsenal.ac.at; anton.haumer@arsenal.ac.at; hansjoerg.kapeller@arsenal.ac.at; franz.pirker@arsenal.ac.at tances, the masses and the inertia can be determined.

The electromagnetic calculation software computes the parameters for the electromechanical model. The electromechanical model can then be applied to the specified operating point or cycle to determine the actual characteristic quantities under these conditions. These characteristic quantities are the currents, power factor, heat losses, efficiency, slip, reactances and characteristic local quantities such as current densities, current coverages, flux densities, etc.

In the next step the thermal behavior of the machine has to be investigated to check whether the thermal limits are exceeded or not. For that purpose a thermal equivalent circuit of the machine, considering the actual cooling conditions, is modeled. The parameters of the thermal equivalent circuit are computed through the geometrical data and the respective material properties. The heat losses determined by the electromechanical model are the heat flow sources for the analysis in the thermal equivalent circuit. A time domain simulation reveals the maximum temperature in the inner parts of the induction machine for a given operating point or cycle. On the one hand, if the thermal limit is exceeded in some parts, the electromagnetic design has to be re-engineered through reducing the heat losses or increasing the thermal capacities, etc. On the other hand, not utilizing the temperature limit due to the insulation class may reveal possible mass and size reductions, if this objective is compatible with the electromagnetic design criteria.

To efficiently compare various machine designs or cooling concepts, a conisitent data exchange between the electromagnetic design software, the electromechanical simulation and the thermal simulation is required. The parameter and data 
handling of the models, simulation and calculation tools presented in this paper are all based on XML (extensible markup language). This data format enables a object oriented description of models and clear structuring of data. For this purpose, an XML library was developed which can be linked either to standard $C$ and Fortran code for the design software or to Modelica code for the electromechanical and thermal simulation tool. The XML library enables reading and writing of scalar and array parameters. The common use of XML data files enables a seamless communication between the electromagnetic design software, the electromechanical simulation and the thermal simulation tools. This way, batch processes can be performed to compare and - in a future version - to automatically optimize certain machine and cooling designs.

In the conventional design process of electric machines, usually, the steady state temperatures may be computed by some simple thermal networks or estimations. The investigation of the impact of a given operating cycle on the actual heat flows and temperatures and the performance and operating behavior of the electromechanical system (the vehicle) can be investigated with the presented approach. The used models and tools represent therefore an innovative step towards the automation of the design process of electric machines.

\section{ELECTROMECHANICAL MODEL}

\subsection{Simulation Tool}

For the simulation of the electromechanical and the thermal model, the integrated modeling and simulation environment Dymola is used. This software is based on the Modelica modeling language [3]. Due to the object oriented structure of Modelica and the comprehensive Modelica Standard Library, covering many physical domains, both the electromechanical and the thermal model can be simulated. The public libraries involved in the presented simulation tools are:

- HeatTransfer - modeling of one dimensional heat transfer with lumped elements

- FluidHeatFlow - modeling of coolant flows as needed for the simulation of electric machines

- Rotational - provides one dimensional, rotational components to model drive trains including friction losses

- Machines - models e.g. permanent magnet synchronous, direct current (DC) and induction machines

Apart from the public libraries, the following in-house developed libraries are used:

- SmartElectricDrives (commercial) - models of quasi stationary and transient electric drives, e.g. permanent magnet synchronous, direct current (DC) and induction machines, batteries, super capacitors, fuel cells and power converters

- SmartPowerTrains (not public) - models of drive train components including brakes, drive resistances, wheels, etc.

\subsection{Induction Machine Model}

The employed electromechanical induction machine model relies on the space phasor theory $[4,5]$. It evaluates the spatial fundamental waves of the electromagnetic quantities in the air gap. This model has, however, no restrictions with respect to the time domain waveforms of the currents and voltages. It is therefore also applicable to inverter supply and transient operating conditions.

In addition to a standard electromechanical model, the following effects are considered:

- Iron losses are modeled by means of a frequency dependent conductor

- Friction losses are considered as a braking torque acting on the shaft

- Speed and current dependent stray losses are taken into account as a braking torque acting on the shaft

For a future implementation, the following effects will be considered by means of a coupled electromechanical/thermal simulation:

- The ohmic resistances of the stator and rotor windings will be modeled temperature dependent; at the moment the electric machine model refers to constant temperatures and the temperature dependent copper losses are adapted in the thermal simulation directly

- If required, the deep bar effect will be taken into account by means of a ladder network of the rotor bars

The model of the electric drive train consists of the electric power supply, the power converter, the electric machine, and the mechanical load including drive resistances, gear (losses), etc. The electric power storage or supply usually is a battery, a super capacitor or a fuel cell. The power converter may be modeled with insulated gate bipolar transistors (IGBTs) or the metal oxide silicon field effect transistors (MOSFETs) including diodes. Alternatively, for inverters with high switching frequencies, instead of modeling the semiconductors a power balance model of the SmartElectricDrives library can be used to consider fundamental wave effects only.

\subsection{Drive Train Model}

An example of an all wheel drive hybrid electric vehicle is depicted in Fig. 1. For the presented hybrid electric vehicle model, components of the Modelica Rotational, the SmartPowerTrains and the SmartElectricDrives [6] library are used. In this model an internal combustion engine (ICE) is directly coupled with the carrier wheel (C) of a planetary gear (PSD). The sun wheel $(S)$ of the planetary gear is connected with the mechanical shaft of the Starter Generator. This starter generator is modeled as permanent magnet synchronous machine. The ring wheel (R) of the planetary gear is connected with the front axle of the vehicle. Both the front and the rear axle model consider two brakes and two tyres including slip model, respectively. Each axle is also directly coupled with an independently controlled induction machine drive. The electric connectors of the electric machines are connected with a battery $(200-280$ V). Each 


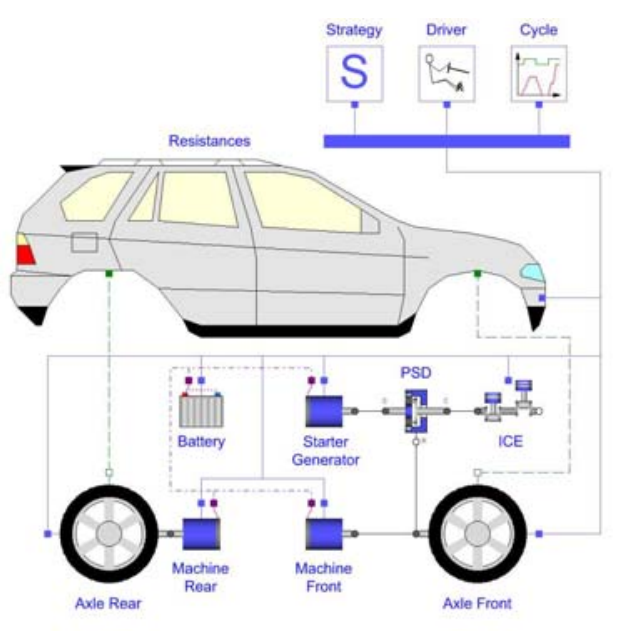

Fig. 1: Simulation model of an all wheel drive hybrid electric vehicle

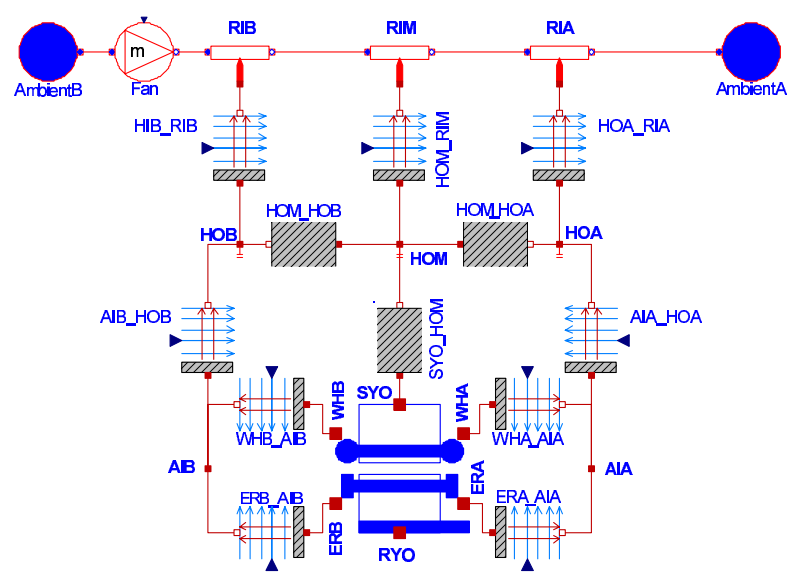

Fig. 2: Thermal equivalent circuit of a surface cooled squirrel cage induction machine

electric machine, however, is equipped with an independent inverter and control unit. Various kinds of drive resistances, such as rolling resistance, aerodynamic resistance and the mass of the vehicle are considered in the Resistances model.

The actual drive cycle to be simulated is loaded by the Cycle model. In this model, the velocity and the gear reference values are put on the bus. The bus, however, holds the relevant signals of the engine and the control system. For simulating the desired drive cycle a Driver model is implemented. This model is realized by proportional plus integral (PI) controller, controlling the gas and break pedal position as well as the changing of the gear.

From the time domain simulation of the electric drive train model, the energy and power flow between energy source, electric machine and mechanical load can be obtained. In addition to that, the losses of the induction machine(s) can provided for the simulation of the thermal model.

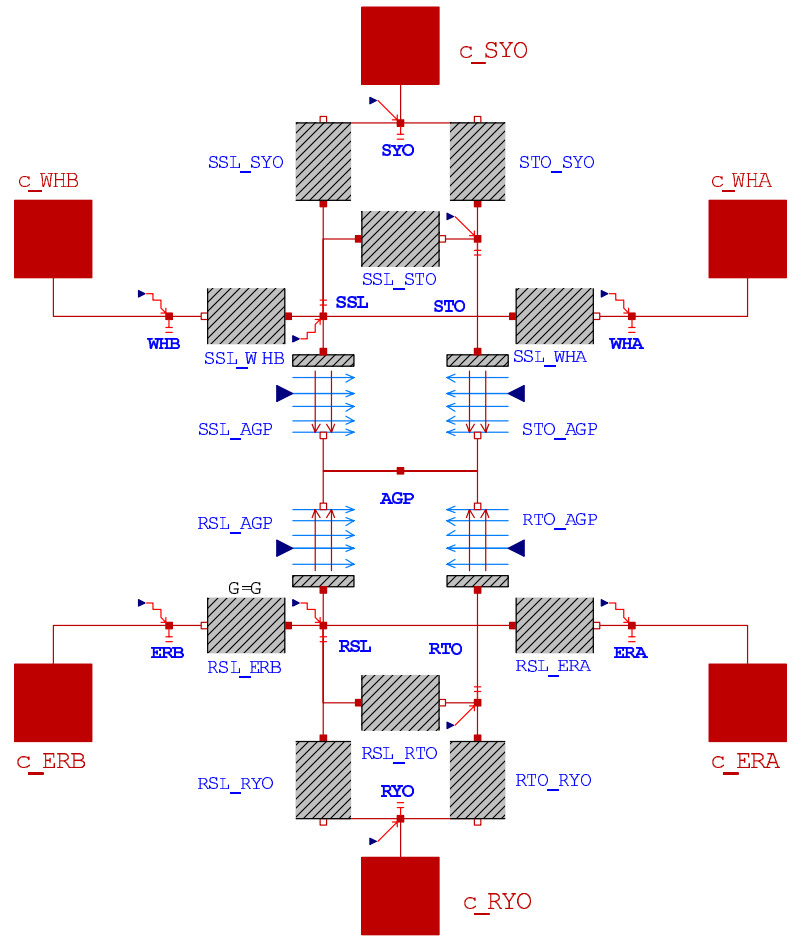

Fig. 3: Active part of an induction machine

\section{THERMAL MODEL}

The thermal behavior of an electric machine is modeled by a thermal network model including cooling circuit (Fig. 2). The thermal network model of the machine consists of the housing parts (end shields, shaft, housing, etc.) and the active part which is depicted in Fig. 3. The active part of the machine comprises the iron core and the windings of the stator and the rotor. A summary of abbreviations of the thermal nodes incorporated in the thermal model of the electric machine is shown in Tab. 1. For the simulation of such a thermal model, the components for modeling the coolant flow, the heat conduction, the heat storage and the loss sources are required $[7,8]$. To study the thermal behavior and the optimal thermal utilization of the machine, models of various cooling concepts can be investigated. For the following, commonly used cooling concepts, thermal models are developed:

- $T E F C$ - totally enclosed fan cooled

- $T E W C$ - totally enclosed water cooled

- $O C V$ - open circuit ventilated

The library for modeling the coolant flow has to be able to handle mixing and splitting of coolant flows. Furthermore, revising the direction of the coolant flow has to modeled appropriately. In the applied thermal model, the medium is considered to be incompressible and the medium properties are considered to be constant. These assumptions, however, are usually fulfilled for cooling circuits of electric machines and lead therefore to an efficient simulation. For the modeling of the thermal network the Modelica FluidHeatFlow and the HeatTransfer library are used. 
Table 1: Thermal nodes of the induction machine model

\begin{tabular}{ll} 
node & area \\
\hline RIA & ribs, A-side \\
RIB & ribs, B-side \\
RIM & ribs, middle area \\
HOA & housing, A-side \\
HOB & housing, B-side \\
HOM & housing, middle area \\
SYO & stator yoke \\
SSL & stator slots \\
STO & stator teeth \\
WHA & winding head, A-side \\
WHB & winding head, B-side \\
AGP & air gap \\
ERA & end ring, A-side \\
ERB & end ring, B-side \\
RSL & rotor slots \\
RTO & rotor teeth \\
RYO & rotor yoke \\
AIA & inner air of the end shield, A-side \\
AIB & inner air of the end shield, B-side
\end{tabular}

The determination of the parameters of the thermal model requires a deep understanding of thermodynamic effects. In practice, these parameters can either be computed by a preprocessing tool, or are included in the initialization of the time domain simulation.

The thermal model (Fig. 2-3) of the investigated TEFC induction machine is designed in the style of an electric network. Such a network consists of the following components:

- Nodes - regions of constant temperature; the potential of a node represents the absolute temperature of that node; the SI unit of the absolute temperature is $\mathrm{K}$

- Loss sources - are equivalent to a current source in an electric circuit; there are loss sources being precalculated losses that have to be corrected by the actual temperature of the corresponding node in order to consider copper losses correctly; other loss sources such as iron losses do not need a temperature dependent correction; the SI unit of the heat losses is W

- Thermal resistors - regions of heat conduction; in the presented thermal equivalent circuit only heat conduction and convection are considered whereas the impact of heat radiation is neglected; the SI unit of a thermal resistance is $\mathrm{K} / \mathrm{W}$

- Thermal conductors - reciprocal of a thermal resistor; the SI unit of a thermal conductance is W/K

- Thermal capacitors - represent the ability of storing heat energy in a certain region; the SI unit of a thermal capacitance is $\mathrm{Ws} / \mathrm{K}$; for a stationary analysis this components do not have to be considered

\section{SIMULATION AND MEASUREMENT RESULTS}

The following simulation results refer to $18.5 \mathrm{~kW}$ TEFC four pole induction machine operated at continuous duty with intermittent periodic loading (duty cycle S6, which is a standard duty cycle for electric machines). In this duty cycle, the induction machine is permanently energized (magnetizing current), and the load torque is alternating between no load (6 min) and $140 \%$ load (4 min) periodically. During the no load period a fraction of the nominal stator copper and the nominal iron losses occur, even, although the induction machine is not mechanically loaded. For $140 \%$ load period, approximately twice the nominal stator copper losses and the nominal iron losses occur. The investigated induction machine is a standard industrial motor which was used for developing and testing the thermal simulation tool.

The simulated temperatures of the stator slots and teeth and the stator winding heads on the drive end and the non drive end side are shown in Fig. 4. The temperatures of the rotor slots and teeth as well as the temperature of the rotor end ring with respect to the drive end and the non drive end are depicted in Fig. 5. The less critical yoke and housing temperature are shown in Fig. 6. All depicted temperature rises refer to an ambient temperature of approximately $30^{\circ} \mathrm{C}$.

The maximum temperature rise of the stator winding is $85^{\circ} \mathrm{C}$. This peak temperature is reached in both the winding heads. The maximum temperature of the stator slots does not exceed $70^{\circ} \mathrm{C}$ and is therefore $15^{\circ} \mathrm{C}$ less than the temperature rise in the winding heads. Thermally, the stator winding is the most critical part due to the insulation class of the insulation material. For assessing the average stator winding temperature, the contributing temperatures (stator slot, both winding heads) have to be averaged taking the respective mass fractions into account.

For the investigated induction machine with die cast rotor, the rotor temperature is less critical due to the missing insulation of the rotor aluminum. The simulation shows a peak temperature rise of approximately $95^{\circ} \mathrm{C}$ in the rotor slots and teeth, and a peak temperature rise of approximately $90^{\circ} \mathrm{C}$ in the end rings and rotor yoke.

Some measurement results are also obtained from the investigated induction machine [9]. The temperature of the stator slot and tooth as well as the winding head temperatures on both side are depicted in Fig. 7. The comparison of these curves with the simulation results of Fig. 4 show a very good match, however.

Measured temperatures from the rotating parts and the air gap are not available. Nevertheless, the rotor temperatures may be critical in some practical application. In such cases it is then useful to monitor the rotor temperature through mathematical machine models [10].

\section{COOLING SYSTEM}

The stator yoke and housing temperature (Fig. 6) are not critical with respect to the material properties. The housing temperature with respect to the cooling temperature, and the speed of the coolant flow determine the heat transport 


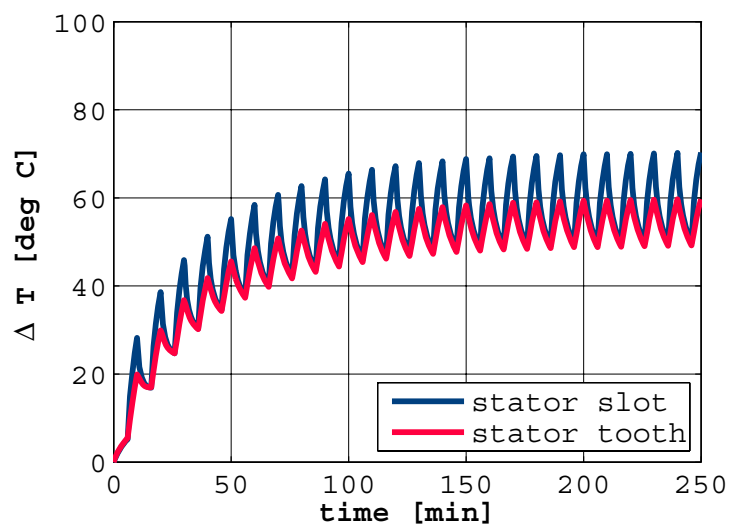

(a) Stator slot and stator tooth temperature

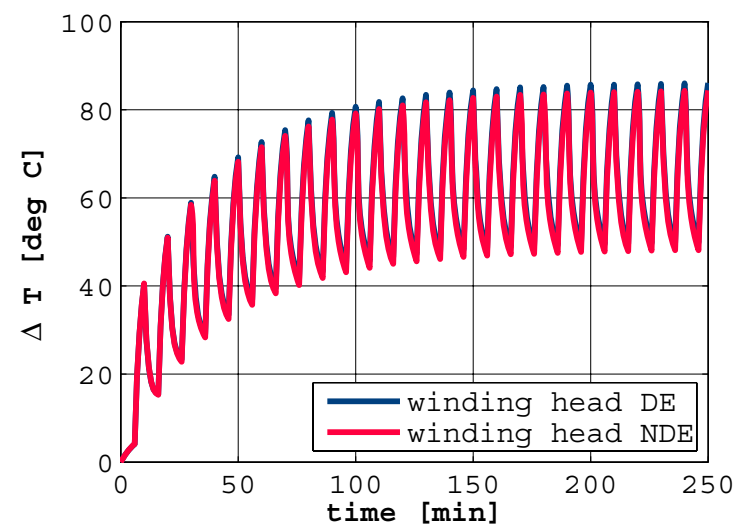

(b) Temperature of stator winding heads of the drive end and non drive end side

Fig. 4: Simulated stator temperatures for duty cycle S6

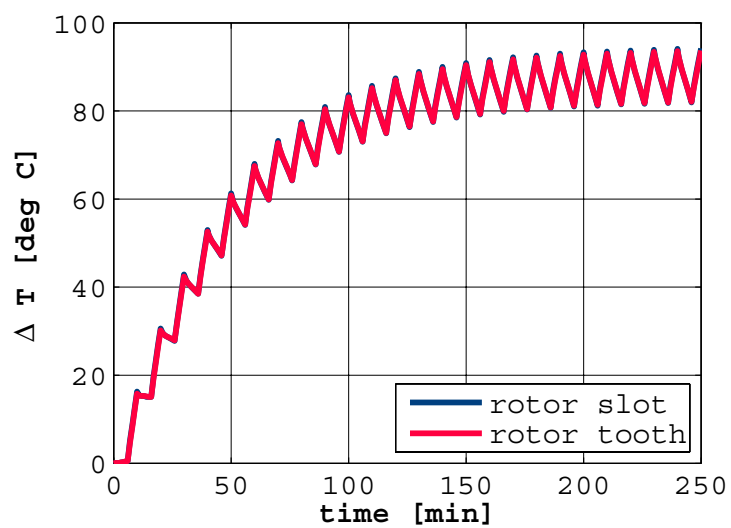

(a) Rotor slot and tooth temperature

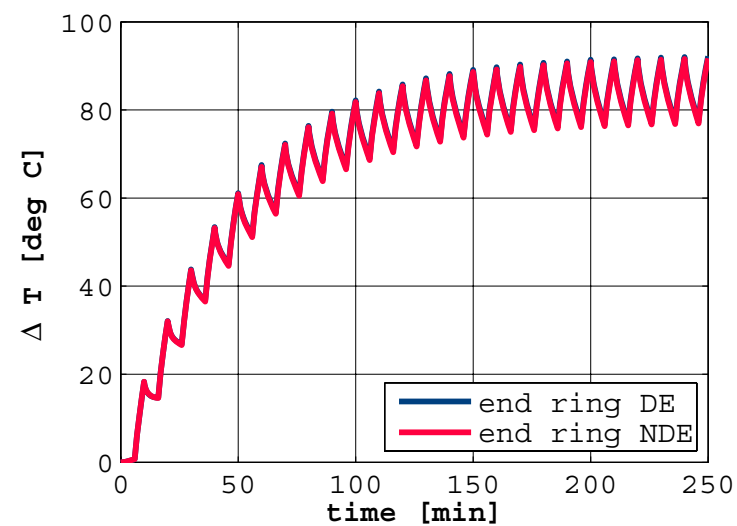

(b) Temperature of the rotor end rings of the drive end and non drive end side

Fig. 5: Simulated rotor temperatures for duty cycle S6

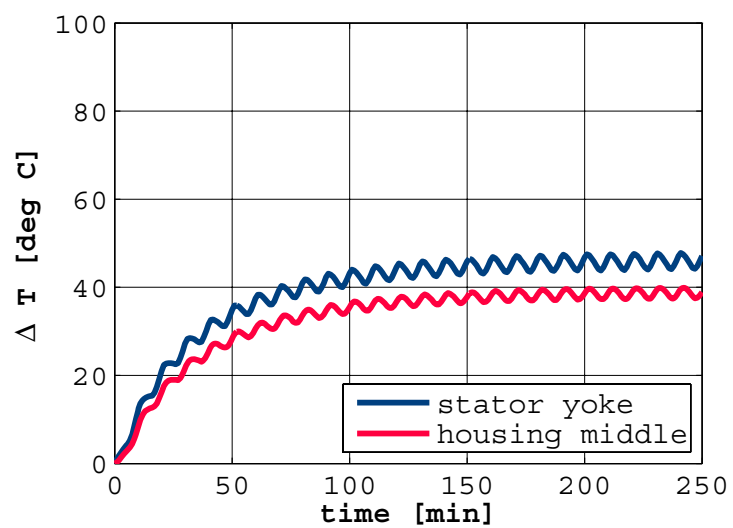

(a) Stator yoke and housing temperature

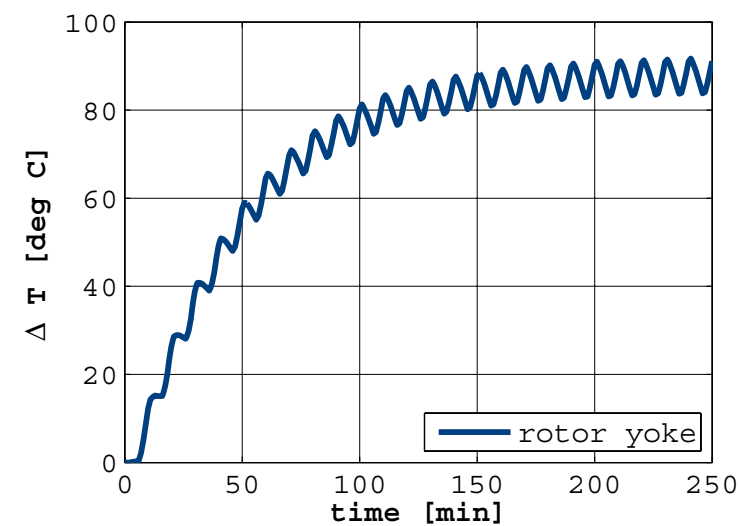

(b) Rotor yoke temperature

Fig. 6: Simulated yoke and housing temperatures for duty cycle S6 


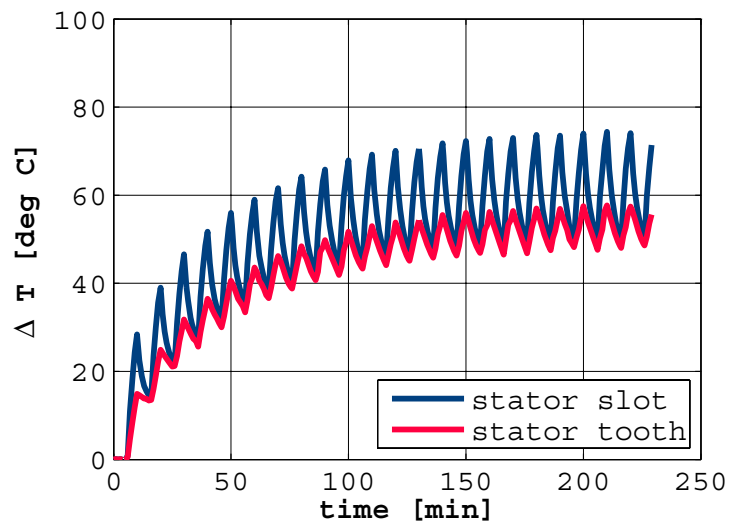

(a) Stator slot and stator tooth temperature

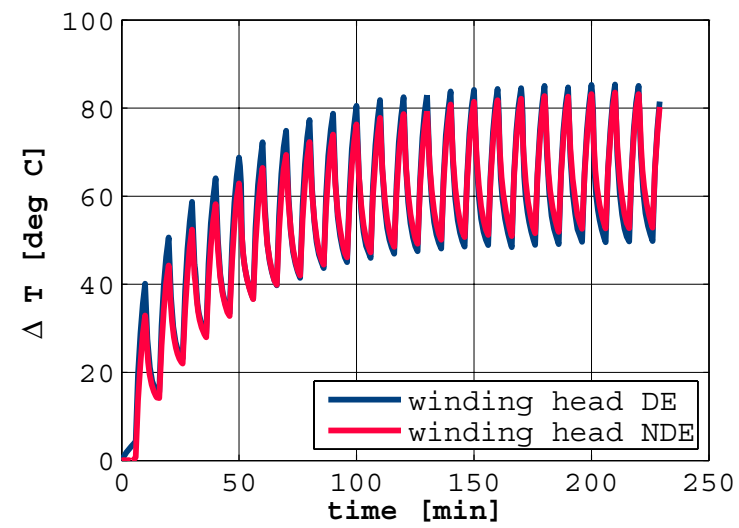

(b) Temperature of stator winding heads of the drive end and non drive end side

Fig. 7: Measured stator temperatures for duty cycle S6

through the coolant. The investigated TEFC machine has a cooling fan mounted on the non drive end of the shaft. The air flow is thus dependent on the actual shaft speed. In the low speed range, the cooling effect of the machine is therefore less than in the nominal speed range.

For a certain electric machine, the following measures may be taken to improve the performance of such a drive:

- With a separately driven cooling fan the speed of the cooling fan can be controlled independently of the drive speed; this improves the cooling performance in the the low speed range

- A better cooling fan with a higher air flow may defuse critical thermal situations of the machine

- Using a water cooling system instead of an air cooling system will also improve the drive performance, since the specific heat capacity of water is higher than the heat capacity of air

\section{CONCLUSIONS}

The design and thermal simulation process of an induction machine for traction applications in electric and hybrid electric vehicles is presented. The design process incorporates the electromagnetic design, an electromechanical simulation considering the actual load profile or cycle and a thermal simulation for the determination of the maximum temperatures in the induction machine.

For a $18.5 \mathrm{~kW}$ totally enclosed fan cooled induction machine, simulated and measured temperatures for a continuous duty with intermittent periodic loading (duty cycle S6) are presented. The matching simulation and measurement results emphasize the potentialities of the developed simulation tools in the design process of induction machines for electric or hybrid electric vehicles.

\section{REFERENCES}

[1] K. Vogt and G. Müller, Elektrische Maschinen, Berechnung rotierender elektrischer Maschinen (in German). Berlin: VEB Verlag Technik, 2 ed., 1974.

[2] B. Chalmers and A. Williamson, A.C. Machines Electromagnetics and Design. New York: Research Studies Press Verlag, 1991.

[3] P. Fritzson, Principles of Object-Oriented Modeling and Simulation with Modelica 2.1. Piscataway, NJ: IEEE Press, 2004.

[4] P. Kovacs, Transient Phenomena in Electrical Machines. Budapest: Akademiai Kiado Verlag, 1984.

[5] C. Kral and A. Haumer, "Modelica libraries for dc machines, three phase and polyphase machines," Modelica Conference, pp. 549-558, 2005.

[6] H. Giuliani, C. Kral, J. Gragger, and F. Pirker, "Modelica simulation of electric drives for vehicular applications . the smart drives library," ASIM, 2005.

[7] C. Kral, A. Haumer, and M. Plainer, "Simulation of a thermal model of a surface cooled squirrel cage induction machine by means of the SimpleFlow-library," Modelica Conference, pp. 213-218, 2005.

[8] G. Rippar and B. Zechmeister, "Simulation of networks of heat sources (in German)," Elin-Zeitschrift, vol. 1, no. Heft 1, 1971.

[9] C. Kral, A. Haumer, M. Haigis, H. Lang, and H. Kapeller, "Comparison of measurements with a cfd analysis and a thermal equivalent circuit model of a tefc induction machine," XVII International Conference on Electrical Machines ICEM 2006, Greece, 2006.

[10] C. Kral, T. Habetler, R. Harley, F. Pirker, G. Pascoli, H. Oberguggenberger, and C.-J. Fenz, "Rotor temperature estimation of squirrel-cage induction motors by means of a combined scheme of parameter estimation and a thermal equivalent model," IEEE Transactions on Industry Applications, vol. 40, pp. 1049-1057, July/August 2004. 


\section{BIOGRAPHIES}

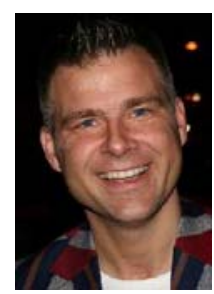

Christian Kral received the Dipl.-Ing. and $\mathrm{Ph} . \mathrm{D}$. degrees from the Vienna University of Technology, Vienna, Austria, in 1997 and 1999, respectively. From 1997 to 2000, he was a Scientific Assistant at the Institute of Electrical Drives and Machines, Vienna University of Technology. Since 2001, he has been with Arsenal Research (Österreichisches Forschungsund Prüfzentrum Arsenal Ges.m.b.H.), Vienna, Austria. From January 2002 until April 2003 he was on a sabbatical as a Visiting Professor at the Georgia Institute of Technology, Atlanta. His research activities are focused on diagnostics and monitoring techniques, machine models, and the simulation of faulty machine behavior. Christian Kral is IEEE Senior Member as well as member of the Austrian Electrotechnical Association OVE and the Modelica Association.

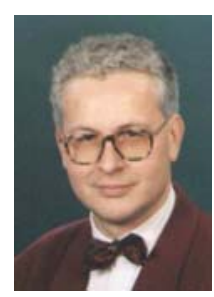

Anton Haumer was born in 1957 in Vienna. He received the Dipl.-Ing. degree in electrical engineering from Vienna University of Technology, Austria, in 1981. He worked for 15 years at ELIN Union AG, later VA Tech ELIN $\mathrm{EBG}$, in various positions in the field of electric drives, especially development and design of electric motors. 1997 he achieved the license "Technical Consulting - Electrical Engineering". After some more years of experience in the field of electric measurement, sensors and automation, as well as power supply systems he began to work as a self-employed technical consultant. He also has several years of experience as trainer and teacher. Since 2004 he is associated with Arsenal Research, Vienna, Austria. His main interests are development and simulation of electric drives. As a member of the Modelica Association, he developed several Modelica Libraries for the simulation of electric drives and acts as the Program Chair of the 5th International Modelica Conference 2006.

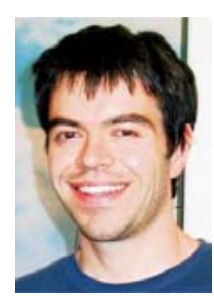

Hansjörg Kapeller was born in 1978 in Italy. He received the Dipl.-Ing. degree in electrical engineering from Vienna University of Technology, Vienna, Austria, in 2004. Since October 2004, he has been an engineer of the business unit Monitoring, Energy and Drive Technologies with Arsenal Research, Vienna, Austria. His main research interests are monitoring of faulty induction machines, simulation and control of electric drives.

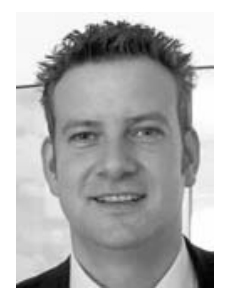

Franz Pirker was born in 1968. He received the Dipl.-Ing. degree in electrical engineering from Vienna University of Technology, Vienna, Austria, in 1997. Since 1999, he has been the head of the business unit Monitoring, Energy and Drive Technologies, arsenal research (Österreichisches Forschungsund Prüfzentrum Arsenal Ges.m.b.H.), Vienna,

Austria. In this area, the main research topics are alternative drive concepts like hybrid electric vehicles, energy conversion and monitoring of electrical drives and machines. 